\title{
Successful endoscopic suturing of esophageal perforation after surgical suturing failure
}

Esophageal perforations still present a life-threatening situation. Although stent insertion and clips have a proven high success rate, some perforations are extremely challenging and persist despite several attempts using different closure techniques. Recently a new endoscopic suturing system (OverStitch; Apollo Endosurgery Inc., Austin, Texas, USA) has been introduced for full-thickness tissue approximation. We report two cases of successful suturing of an esophageal perforation using the OverStitch device, after the failure of surgical suturing.

The first patient was an 87-year-old woman with a wide perforation $(35 \mathrm{~mm}$ long $\times 25 \mathrm{~mm}$ ) of the mid-esophagus due to Boerhaave syndrome. She was initially treated surgically but the leak reappeared within the first postoperative week. Closure was attempted endoscopically with hemostatic clips (Instinct Clips; Cook Medical Inc., Bloomington, Indiana, USA), but this also failed. After previous clips had been removed, the margins of the defect were cauterized using argon plasma and three interrupted sutures were applied using the OverStitch device ( $\vee$ Video 1 ). Subsequent contrast medium showed no leaks.

Follow-up examination with contrast medium (first post-treatment week), showed a small persistent leak. The leak was smaller than the initial defect (about $20 \mathrm{~mm}$ long $\times 20 \mathrm{~mm}$ ). Three interrupted sutures were applied using OverStitch, and contrast medium showed no residual leak. A metal fully covered stent, $100 \times 14$ mm (Niti-S; Taewoong Medical, Gyeonggi-do, South Korea) was finally placed. After 1 month, the stent was removed and the defect had closed successfully ( $\triangleright$ Fig. 1 ).

The second case was a 73-year-old woman with an esophageal perforation caused by the impaction of a turkey bone. The defect was initially treated by surgery but the contrast medium 2 weeks after

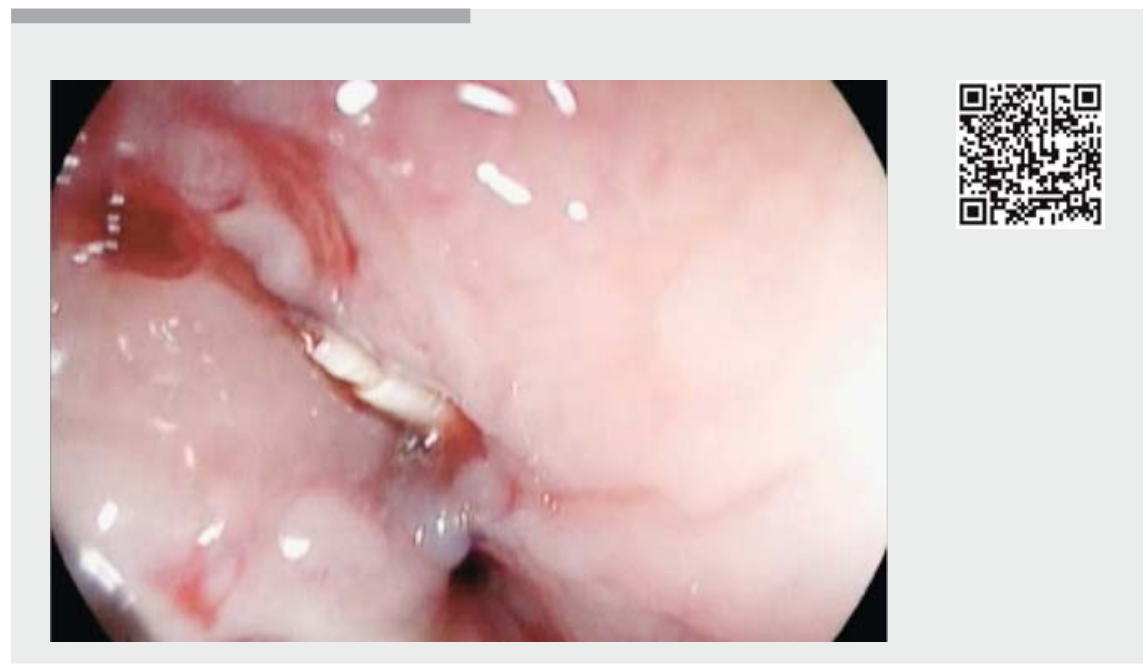

Video 1 Closure of an esophageal perforation using the OverStitch device (Apollo Endosurgery Inc., Austin, Texas, USA). A Large mid-esophageal defect was present after a failed attempt at surgical closure. After complete cleaning of the para-esophageal cavity, the margins of the defect were cauterized using argon plasma before suturing. Then, interrupted sutures were applied using the OverStitch device, and the closure of the esophageal defect was finally achieved.

surgery showed a persistent leak. Endoscopy revealed a $30 \mathrm{~mm}$ long $\times 25 \mathrm{~mm}$ perforation in the mid-esophagus. The margins were cauterized using argon plasma. Three interrupted sutures were applied using the Apollo OverStitch. A metal fully covered stent, $100 \times 20 \mathrm{~mm}$ (Niti-S; Taewoong Medical) was placed to cover the suture. However, post-treatment radiology at 1 week showed a smaller persistent esophageal defect. After removal of the stent, the defect was successfully closed with three interrupted sutures at a second session, and a metal fully covered stent, $100 \times 22 \mathrm{~mm}$ (Niti-S; Taewoong Medical) was placed. Closure was radiologically confirmed 4 weeks later. Although larger series are needed to confirm our initial experience, these cases suggest that OverStitch is a promising endoscopic device for closing large esophageal perforations, even after surgical failure.

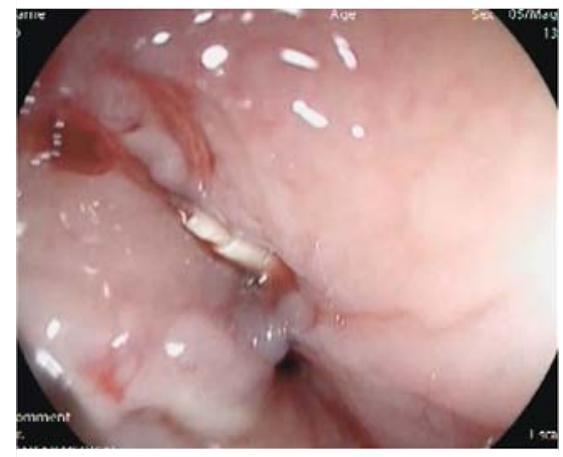

- Fig. 1 Closure of the esophageal perforation 1 month after suturing using the OverStitch device (Apollo Endosurgery Inc., Austin, Texas, USA). 
None

The Authors

Roberta Maselli ${ }^{1,2}$, Edi Viale ${ }^{1}$, Lorella Fanti ${ }^{1}$, Pier Alberto Testoni ${ }^{3}$

1 Gastroenterological and Endoscopic Unit, Division of Experimental Oncology, IRCCS San Raffaele Scientific Institute, Milan, Italy

2 Surgical Sciences Department, Sapienza University of Rome, Rome, Italy

3 Gastroenterological and Endoscopic Unit, Division of Experimental Oncology, Vitasalute San Raffaele University, Milan, Italy
Corresponding author

Roberta Maselli, MD

Gastroenterological and Endoscopic Unit, Division of Experimental Oncology, IRCCS

San Raffaele Scientific Institute, via

Olgettina, Milan 20132, Italy

Fax: +39-33-38007368

roberta.maselli.md@gmail.com

\section{Bibliography}

DOI https://doi.org/10.1055/s-0043-108647

Published online: 5.7.2017

Endoscopy 2017; 49: E202-E203

(c) Georg Thieme Verlag KG

Stuttgart · New York

ISSN 0013-726X

\section{ENDOSCOPY E-VIDEOS}

https:/|eref.thieme.de/e-videos

回回 Endoscopy E-Videos is a free access online section, reporting 回和: on interesting cases and new techniques in gastroenterological endoscopy. All papers include a high quality video and all contributions are freely accessible online.

This section has its own submission website at

https://mc.manuscriptcentral.com/e-videos 